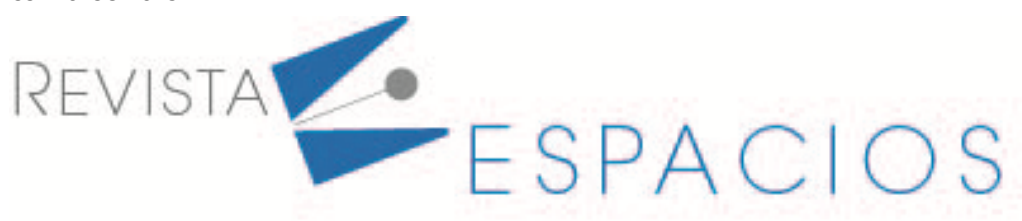

Vol. 41 (50) $2020 \cdot$ Art. 4

\title{
Sistema loT para la monitorización y análisis de niveles de ruido
}

\section{IoT system for monitoring and analyzing noise levels}

\author{
CHANCHÍ, Gabriel E. ${ }^{1}$ \\ OSPINA, Manuel A. ${ }^{2}$ \\ SABA, Manuel ${ }^{3}$
}

\begin{abstract}
Resumen
Partiendo de las limitaciones de los dispositivos de medición de nivel de ruido, en cuanto al almacenamiento limitado, la monitorización en tiempo real y el análisis de los datos capturados, este artículo propone como aporte un sistema loT para la monitorización y análisis de niveles de ruido, el cual se articula dentro de la arquitectura convencional de loT y pretende servir de referencia para ser usado en auditorias acústicas.
\end{abstract}

Palabras clave: arquitectura iot, sistema de monitoreo de ruido, sonómetro, sistema de bajo costo.

\begin{abstract}
Based on the limitations of noise level measurement devices, in terms of limited storage, real-time monitoring and analysis of captured data, in this paper we propose as a contribution an loT system for monitoring and analysis of noise levels, which is framed within the conventional architecture of loT and is intended to serve as a reference for use in acoustic audits.

key words: internet of things, noise monitoring system, sound level meter, low cost system.
\end{abstract}

\section{Introducción}

La contaminación acústica, también conocida como ruido ambiental, es la propagación del ruido con impactos variables sobre la vida humana (Basner et al., 2014). La Organización Mundial de la Salud reconoce el ruido como uno de los principales peligros ambientales para la salud física y mental (Marouf et al., 2020; World Health Organization, 2018). Una elevada exposición al ruido por tiempos prolongados puede generar altos niveles de estrés, efectos cardiovasculares y una mayor incidencia de enfermedad coronaria entre otros (Begou et al., 2020a; Khan et al., 2020; Recio et al., 2018; Thacher et al., 2020). Algunas de las principales fuentes de ruido en las áreas residenciales incluyen música alta, transporte (tráfico vehicular, ferrocarril, aviones, etc.), mantenimiento del césped, industria de la construcción, generadores eléctricos, explosiones, entre otros (Ruparel et al., 2013). Adicionalmente, la mala planificación urbana puede dar lugar a un aumento de la

\footnotetext{
1 Profesor. Programa Ingeniería de Sistemas. Facultad de Ingeniería. Universidad de Cartagena. gchanchig@unicartagena.edu.co 2 Profesor. Programa Ingeniería de Sistemas. Facultad de Ingeniería. Universidad de Cartagena. mospinaa@unicartagena.edu.co

3 Profesor. Programa Ingeniería Civil. Facultad de Ingeniería. Universidad de Cartagena. msaba@unicartagena.edu.co
} 
contaminación acústica, como por ejemplo el caso de los edificios industriales a lado de los edificios residenciales (Montes et al., 2020).

La sensibilización, la planificación y el control son herramientas clave para enfrentar este problema (Shakya et al., 2019). Específicamente, este trabajo se enfoca en los sistemas de control urbano. Las fuentes de ruido no son constantes y su percepción depende de muchos factores. Varios indicadores del nivel de ruido equivalente durante el día y durante la noche han sido desarrollados alrededor del mundo como reporta (Khan et al., 2018). Los sistemas de monitoreo del ruido, que a menudo son acoplados con los sistemas de monitoreo de calidad de aire (Khan et al., 2018), son generalmente costosos y generan costos de mantenimiento altos, por lo que son sistemas comunes en las grandes ciudades de los países desarrollados, (Khan et al., 2020). Sin embargo, en las ciudades de los países en desarrollo los sistemas de medición a menudo son puntuales, tienen limitaciones en el almacenamiento, operados manualmente, y no están conectados a una red de monitoreo en tiempo real, (Sieber et al., 2018; Zambrano-Monserrate \& Ruano, 2019). A tal propósito, en la última década se han desarrollado nuevas generaciones de sensores inalámbricos comerciales, los cuales son considerados como una herramienta válida de monitoreo para la evaluación del ruido del tráfico vehicular. Aunque presenten en muchos casos menor precisión en la medición, tienen costos sensiblemente menores. Esto permite crear una red capilar de medición, que puede ser acoplada a un centro de control, análisis y visualización de los datos en tiempo real, generando sistemas de alarma y de alerta temprana, garantizando un mejor control de la contaminación acústica en ámbito urbano, (Marouf et al., 2018; Marouf et al., 2020). Este tipo de red, acoplada a un sistema de medición de referencia, podría servir para bajar los costos e incentivar las autoridades competentes de los países en desarrollo a implementar un sistema de monitoreo urbano de contaminación acústica.

A partir de lo anterior, en este artículo se propone como aporte un sistema loT para la monitorización y análisis de niveles de ruido en auditorias acústicas, el cual está articulado dentro de las cuatro capas de la arquitectura convencional de loT (captura, almacenamiento, análisis y visualización) (Quiroga et al., 2017). De este modo el sistema propuesto pretende solventar las limitaciones de los instrumentos de medida empleados en las auditorias acústicas, en lo referente al almacenamiento limitado, el acceso restrictivo a los datos, el seguimiento remoto y la aplicación de algoritmos de aprendizaje supervizado y no supervizado a los datos capturados. Para la construcción del sistema, se hizo uso de un sonómetro bluetooth en la capa de captura, de tal modo que el sistema amplía las funcionalidades provistas por este al posibilitar el almacenamiento de los datos, el seguimiento en tiempo real y la aplicación de algoritmos de clustering. El sistema propuesto puede servir de referencia en cuanto a construcción de sistemas de monitoreo de diferentes variables ambientales, aprovechando las ventajas provistas por protocolos de comunicación tales como bluetooth LE.

Para ampliar la argumentación en cuanto a la arquitectura loT propuesta, a continuación se presentan un conjunto de trabajos relacionados que se tuvieron en cuenta para el desarrollo del presente proyecto.

En (Leiva \& Higuera, 2019) se presenta un sistema loT para la monitorización de ruido en entornos cerrados, el cual fue construido haciendo uso de la plataforma Arduino y los sensores de ruido ky-038. Los datos capturados por la plataforma Arduino son convertidos a la escala de decibeles y enviados mediante el puerto serial a un computador donde los datos son visualizados. El sistema no cuenta con la posibilidad de realizar la monitorización remota en tiempo real de los niveles de ruido obtenidos a partir de los sensores, ni tampoco permite el almacenamiento y procesamiento de los datos mediante algoritmos de analítica.

En (Suárez et al., 2017) se presenta el diseño de una herramienta de medición de ruido basada en el uso de plataformas tipo SBC (Single Board Computer) tales como Arduino y Raspberry PI. El sistema propuesto hace uso de sensores de ruido $D$ fRobot 0034 para la captura de los niveles de ruido, los cuales son obtenidos por la tarjeta Arduino y enviados a la plataforma Raspaberry PI, desde la cual los datos son enviados a una base de datos en la 
nube, donde son consultados los datos mediante una aplicación web. La herramienta propuesta no permite el monitoreo remoto de los datos, ni tampoco cuenta con un módulo para el análisis de los datos capturados.

En (Hernández \& Barriere, 2017) se presenta el desarrollo de un sistema para la monitorización de parámetros ambientales en unidades de cuidados intensivos neonatales. El sistema propuesto fue construido haciendo uso de la plataforma de hardware libre y un conjunto de sensores compatibles con esta plataforma, los cuales permiten medir los parámetros de temperatura, humedad, intensidad luminosa e intensidad sonora. Para el caso de la medición de la temperatura y la humedad se hace uso del sensor DHT11, mientras que para la medición del nivel de ruido e intensidad luminosa se emplean los sensores KY-038 y KY-018 respectivamente. Los datos capturados por los sensores son enviados a la tarjeta Arduino y luego transmitidos mediante puerto serial a un computador en donde la información es presentada en una interfaz gráfica desarrollada en Labview. El sistema propuesto no permite la lectura remota de los datos, así como tampoco cuenta con las funcionalidades de almacenamiento y análisis mediante algoritmos de machine learning.

En (Nurjannah et al., 2017) se propone un sistema embebido basado en herramientas de hardware libre como Arduino y el sensor KY-038, para la captura de niveles de ruido, así como el almacenamiento local y la visualización en una pantalla LCD de los datos capturados en tiempo real. El trabajo presenta como aporte la calibración del sensor KY-038 haciendo uso de un sonómetro comercial, sin embargo, el sistema embebido desarrollado no permite el seguimiento remoto, ni hace uso de modelos de analítica para el procesamiento de los datos.

En (Quiroga et al., 2017) se presenta una arquitectura loT basada en herramientas de hardware y software libre para el soporte de agricultura de precisión. La arquitectura propuesta toma en consideración las capas de la arquitectura Lambda: captura de datos, almacenamiento, procesamiento de datos y consulta. En la capa de captura se hace uso de sensores tales como los de temperatura y humedad, en la capa de almacenamiento se emplea la base de datos en la nube Ubidots, en la capa de análisis se hace uso de un clasificador bayesiano y finalmente en la capa de consulta se hace uso de un aplicativo web desarrollado sobre el Frame Work Spark de Java. Si bien este trabajo no toma en cuenta dentro de las variables a medir el nivel de ruido, fue tenido en cuenta las capas de la arquitectura consideradas para el diseño del sistema loT de seguimiento y análisis de niveles de ruido.

Existen además en la literatura diferentes técnicas para la medición de ruido (tanto en ambientes abiertos como cerrados) (Begou et al., 2020b; Hueso et al., 2017; Neitzel et al., 2016; Peng et al., 2018; Senthilkumar et al., 2020; Stoilova et al., 2013), estas técnicas hacen uso de diferentes plataformas para la adquisición, procesamiento y análisis de los datos, evidenciando limitaciones en cuanto al alto costo de los equipos que se deben utilizar, además como se aprecia en los trabajos presentados en esta sección, existen ciertas limitaciones en los sistemas de medición de ruido en cuanto al seguimiento remoto, al almacenamiento y al uso de algoritmos de analítica de datos para el procesamiento de los niveles de ruido capturados. En este sentido, el presente trabajo pretende abordar las anteriores limitaciones en el marco de una arquitectura convencional de loT a 4 capas (captura, almacenamiento, análisis y visualización).

El resto del artículo está organizado de la siguiente manera: en la sección 2 se presenta la metodología empleada para el desarrollo de este trabajo; en la sección 3 se muestra la arquitectura e implementación del sistema loT para la monitorización y análisis de ruido propuesta; en la sección 4 se valida el funcionamiento de la arquitectura loT a partir de un ejemplo de aplicación; finalmente en la sección 5 se muestran las conclusiones y los trabajos futuros derivados de la presente investigación. 


\section{Metodología}

Para el desarrollo de la presente investigación, se consideraron las siguientes 4 fases: caracterización de los niveles de ruido, diseño de la arquitectura del sistema loT, implementación del sistema loT, verificación del funcionamiento (ver Figura 1).

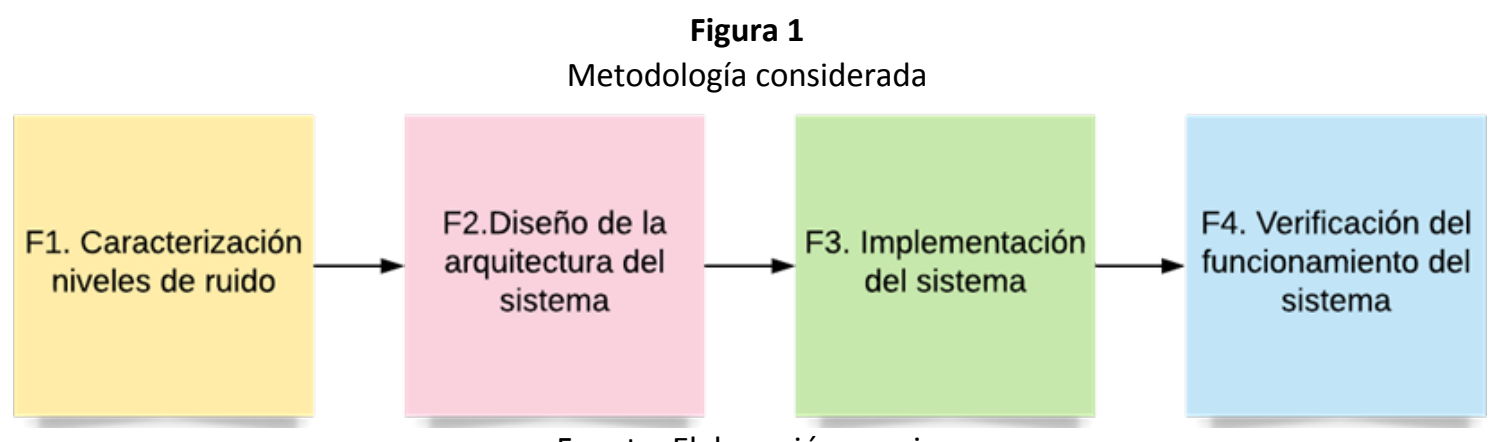

Fuente: Elaboración propia

En la fase 1 se definieron los niveles de ruido que se consideraron para el diseño del sistema loT, teniendo en cuanta una adaptación a los niveles considerados en la normativa colombiana (Suárez et al., 2017). En la fase 2 se diseñaron las capas de captura, almacenamiento, análisis y visualización de la arquitectura del sistema loT, teniendo en cuenta la arquitectura convencional de loT. En la fase 3 se implementó el sistema de seguimiento IoT, haciendo uso de un sonómetro y un conjunto de tecnologías libres para el almacenamiento, análisis y visualización. Finalmente, en la fase 4 se realizó la verificación del sistema implementado a través de un ejemplo de uso del sistema.

\section{Sistema loT propuesto}

En esta sección se presenta la escala de niveles de ruido considerada por el sistema propuesto, así como la arquitectura del sistema y la implementación de este. En este sentido, de acuerdo a la caracterización de los niveles de ruido mencionada en la fase 1 de la metodología, en la Tabla 1 se presentan los niveles de clasificación de ruido en $d B A$ adaptados a partir de lo presentado en (Suárez et al., 2017). Teniendo en cuenta que los sonómetros convencionales no manejan este tipo de clasificación, estos intervalos de niveles fueron empleados por el sistema para la categorización en tiempo real, así como para el análisis de los datos mediante algoritmos de clustering, tales como K-Means.

Tabla 1

Niveles de ruido considerados

\begin{tabular}{|c|c|}
\hline Rango & Categoría \\
\hline Nivel $<=30 \mathrm{dBA}$ & Bajo \\
\hline $30 \mathrm{dBA}<$ Nivel $<=50 \mathrm{dBA}$ & Normal \\
\hline $50 \mathrm{dBA}<$ Nivel $<=75 \mathrm{dBA}$ & Considerable \\
\hline $75 \mathrm{dBA}<$ Nivel $<=100 \mathrm{dBA}$ & Alto \\
\hline $100 \mathrm{dBA}<$ Nivel $<=120 \mathrm{dBA}$ & Muy alto \\
\hline Nivel $>120 \mathrm{dBA}$ & Umbral de dolor \\
\hline
\end{tabular}

Fuente: Elaboración propia

A partir de las clasificaciones de nivel de ruido presentadas en la Tabla 1 se realizó el diseño de la capa de análisis del sistema propuesto. En concordancia con lo anterior, en la Figura 2 se presentan las funcionalidades desarrolladas en cada una de las cuatro capas de la arquitectura del sistema loT propuesto (captura, almacenamiento, análisis y visualización) para la monitorización y análisis del nivel de ruido. En la capa de captura a través de un sonómetro comercial tipo bluetooth los niveles de ruido son capturados, desplegados en la 
pantalla del dispositivo y enviados mediante el protocolo bluetooth a la capa de almacenamiento. En la capa de almacenamiento los datos son capturados mediante una conexión asíncronica realizada a partir de la dirección del dispositivo bluetooth y del código de la característica de envío, de tal modo que a medida que los datos son capturados, se categorizan de acuerdo con los rangos de la Tabla 1 y almacenados en una base de datos no relacional mediante un id propio de cada sesión de captura.

Figura 2

Funcionalidades de las

capas del sistema loT

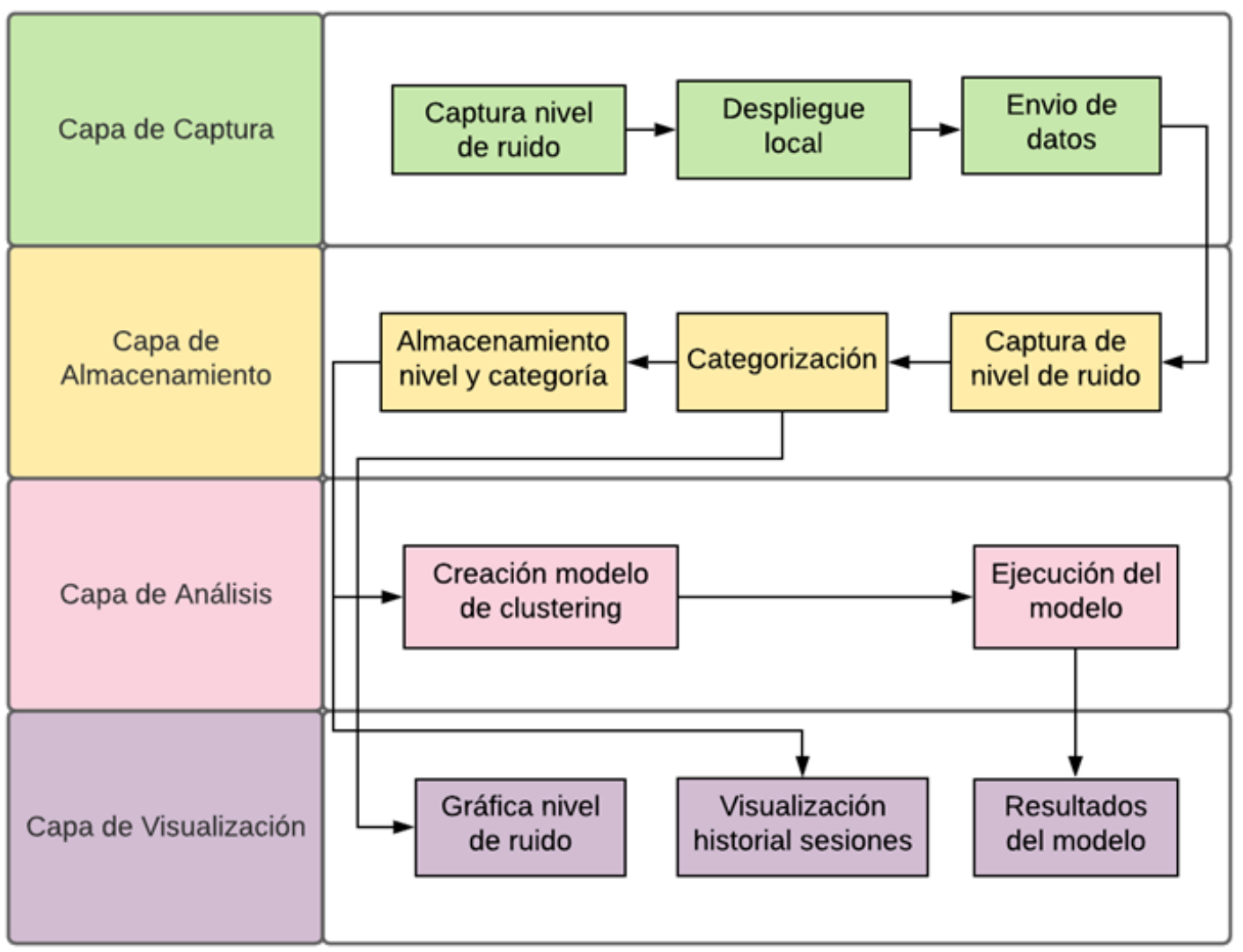

Fuente: Elaboración propia

Una vez los datos son categorizados, en la capa de visualización los datos son presentados a través de una gráfica en tiempo real, al mismo tiempo que es posible realizar una consulta sobre el histórico de los datos almacenados en cada sesión de captura. En la capa de análisis es posible crear modelos de aprendizaje no supervisado, cuyos resultados son presentados en la capa de visualización. Las funcionalidades presentadas en la Figura 2 son implementadas a partir de las tecnologías presentadas en la Figura 3.

En la Figura 3 se muestra como en la capa de captura se hace uso del sonómetro comercial UT353BT el cual permite enviar los niveles de ruido capturados del ambiente, haciendo uso de bluetooth LE mediante un formato de datos definido por el proveedor del equipo, el cual es necesario decodificar a partir de la trama. En la capa de almacenamiento a través de una aplicación desarrollada en Java que usa en segundo plano un script de Python con la librería Bleak, se capturan los datos del nivel de ruido, se categorizan, se presentan de manera gráfica en la capa de visualización usando las librerías XChart y JFreechart y se almacenan por sesiones en la base de datos no relacional jsond $b$ de Java. Del mismo modo los datos almacenados en la base de datos pueden ser consultados en la capa de visualización obteniendo estadísticas básicas como el promedio y la desviación estándar de los niveles de ruido. En la capa de análisis haciendo uso de los datos almacenados en la base de datos y de las funcionalidades provistas por la librería weka de Java se implementa el algoritmo de agrupamiento $K$-means, el 
cual permite obtener un número de terminado de centroides alrededor de los cuales se concentran los datos capturados. Los resultados de la aplicación de los modelos de clustering son presentados en la capa de visualización.

Figura 3

Tecnologías del sistema loT

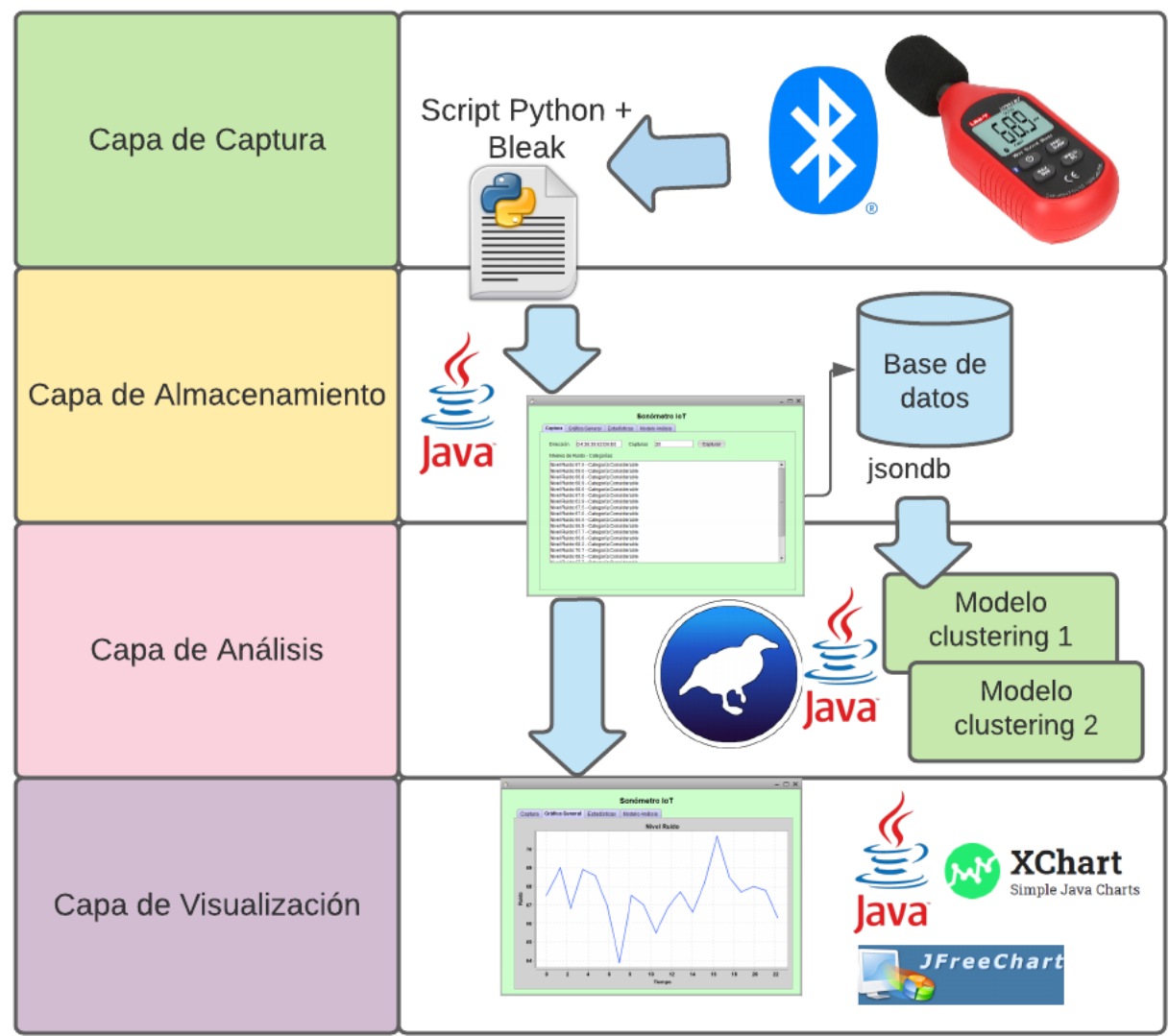

Fuente: Elaboración propia

Teniendo en cuenta las funcionalidades y tecnologías presentadas en las Figuras 2 y 3 , en la Figura 4 se presenta la interfaz principal del sistema loT propuesto, la cual está conformada por las siguientes cuatro pestañas: "Captura”, "Gráfica General”, “Estadísticas” y “Modelo de Análisis".

En la pestaña de "Captura" el usuario del sistema escribe el número de capturas a obtener y la dirección bluetooth del dispositivo, de tal modo que una vez se presiona el botón "Capturar" se establece la comunicación asincrónica con el sonómetro. Los datos que van siendo capturados son categorizados y en el área de texto de la pestaña "Captura" se va presentando en tiempo real el nivel de ruido y la categoría asociada a dicho nivel de acuerdo con los rangos definidos en la Tabla 1, además se van almacenando estos datos en una sesión de captura dentro de la base de datos jsondb. Así, a modo de ejemplo en la Figura 4 se configuró como dirección bluetooth: “D4:36:39:62:D6:E0” y como número de capturas 20 (una captura asincrónica por segundo como mínimo). 
Figura 4

Interfaz principal del sistema loT

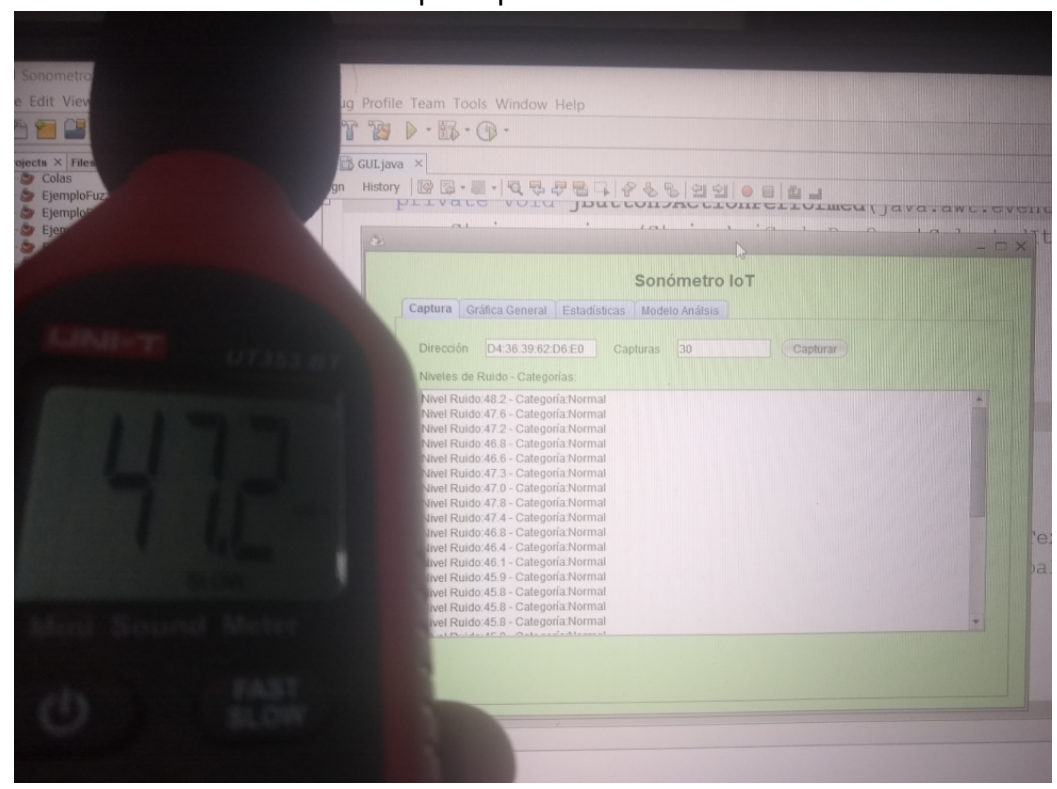

Fuente: Elaboración propia

A medida que los datos son capturados y categorizados, en la pestaña "Gráfica General" se va realizando una gráfica en tiempo real con los niveles de ruido en $d B A s$ en función del tiempo. Esta gráfica se puede realizar gracias a las funcionalidades provistas por la librería XChart de Java (ver Figura 5).

Figura 5

Pestaña gráfica general

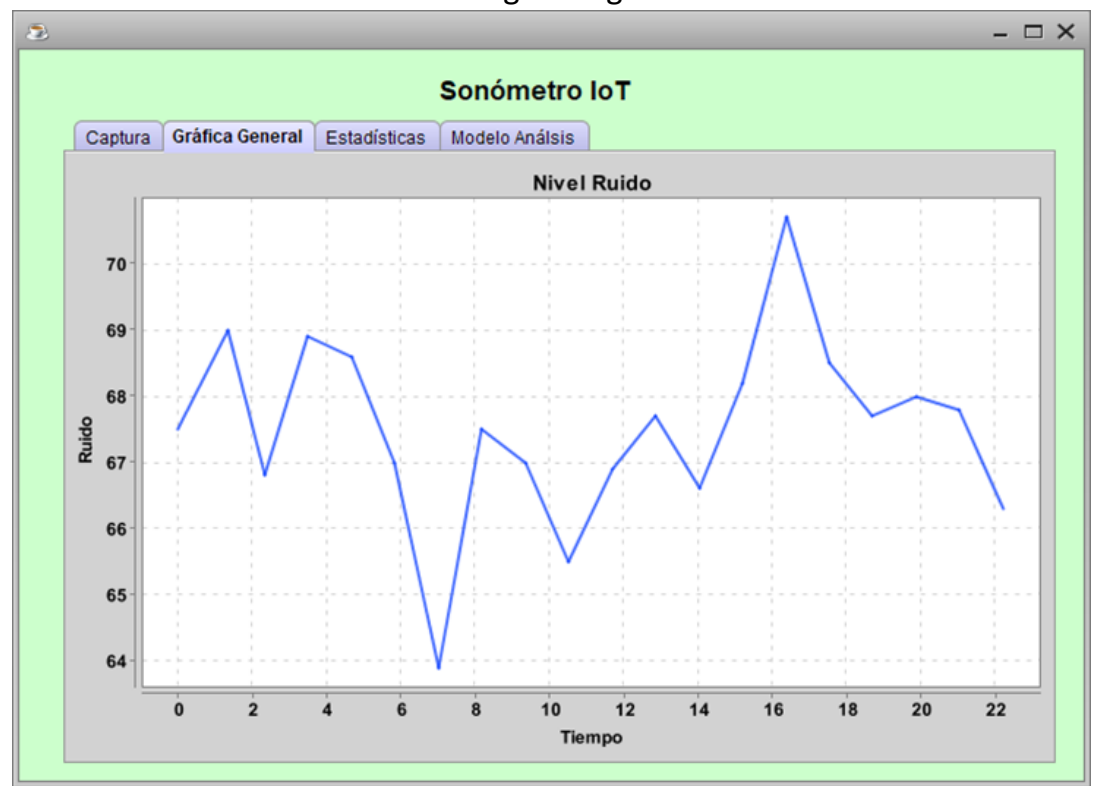

Fuente: Elaboración propia

Por otra parte, en la pestaña "Estadísticas" es posible consultar el histórico de los datos almacenados en una sesión de captura, así como obtener el promedio y la desviación estándar de los niveles de ruido pertenecientes a la sesión de captura. Del mismo modo, en esta pestaña se presenta un diagrama de barras con la distribución de las diferentes categorías definidas en la Tabla 1 y presentes a lo largo de la sesión de captura (ver Figura 6). 
A modo de ejemplo, en la Figura 6 se ha escogido una de las sesiones aleatorias con una duración de 22,324 segundos en la cual se tiene como promedio de los niveles de ruido 55,394 y como desviación estándar de los niveles de ruido 4,70. A partir de la gráfica generada se concluye que existen dos categorías que están presentes en la captura: "Normal” y "Considerable" entre las cuales hay un porcentaje de aparición en la sesión de captura cercano al $20 \%$ y al $80 \%$ respectivamente.

Figura 6

Pestaña estadísticas

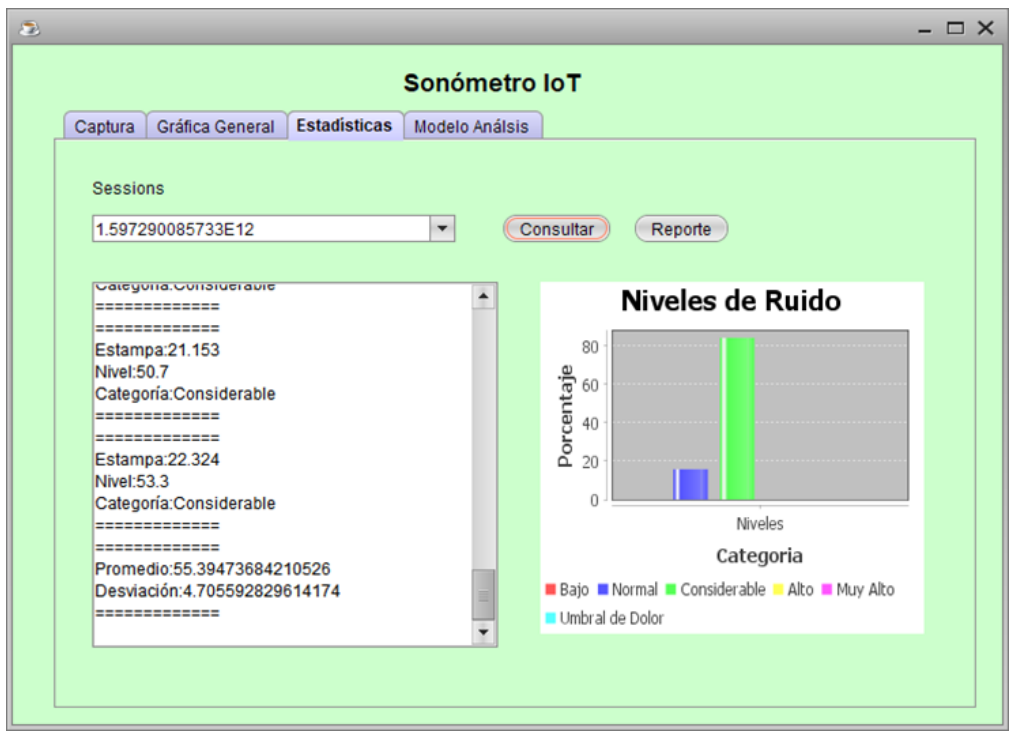

Fuente: Elaboración propia

Por último, en la Figura 7 se muestran los resultados obtenidos al aplicar el algoritmo de clustering K-Means a los datos de la misma sesión de captura considerada en la Figura 6. El algoritmo K-Means se configuró mediante la API de weka para trabajar con los atributos "nivel" y "categoría", de tal modo que como resultado del algoritmo se obtienen dos centroides alrededor de los cuales están concentrados los datos: $C 1=\{$ nivel=56,7375, categoría="considerable" $\}, C 1=\{$ nivel=48,2333, categoría="normal" $\}$. El centroide $C 1$ tiene asociados un total de 16 instancias, mientras que el centroide $\mathrm{C} 1$ cuenta con 3 instancias.

Figura 7

Pestaña modelo análisis

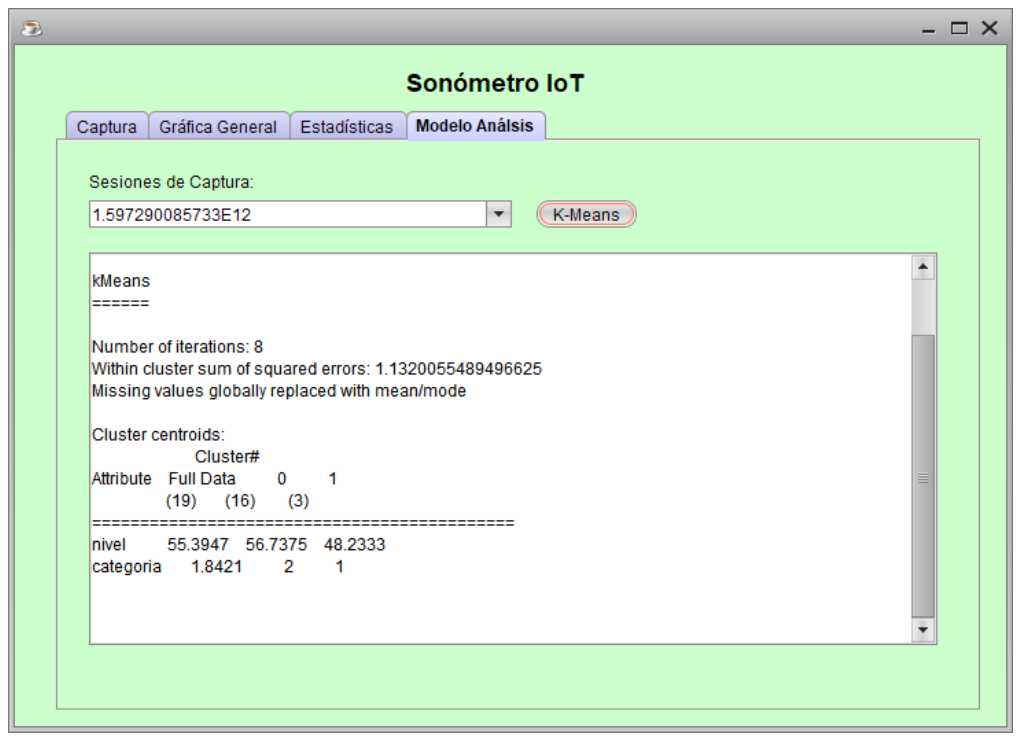

Fuente: Elaboración propia 


\section{Verificación del sistema loT}

Con el fin de verificar el funcionamiento del sistema loT desarrollado e implementado, se realizó una prueba funcional o sesión con 400 capturas tomadas en un edificio residencial de la ciudad de Cartagena de Indias Colombia un fin de semana entre las $8: 00$ pm y 9:00 pm (ver Figura 8). La prueba permitió demostrar el correcto funcionamiento del sistema loT, en donde se obtuvo un valor promedio de nivel de ruido de 56,86 dBA con una desviación estándar de 2,41. Así mismo se obtuvo a partir del reporte de la prueba que el valor mínimo de ruido en la sesión fue de 51,9 dBA, mientras que el valor máximo fue de 71,4 dBA

Figura 8

Verificación del sistema loT

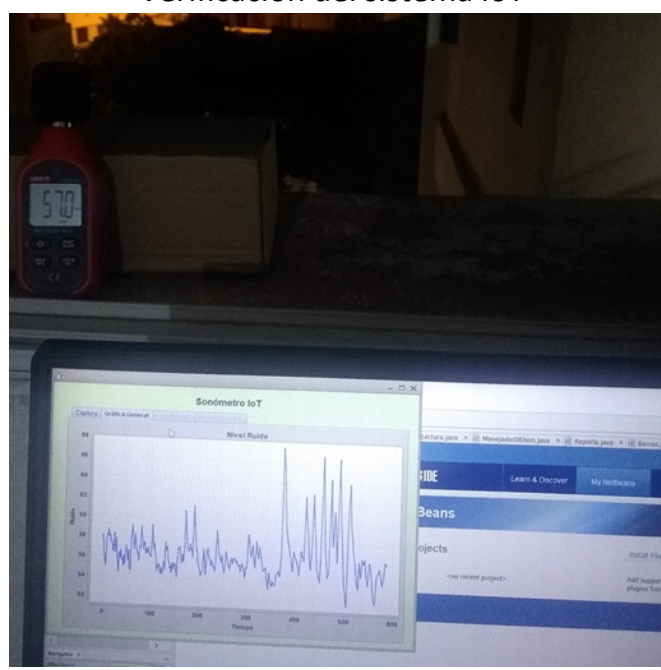

Fuente: Elaboración propia

Finalmente, en la Figura 9 se muestran los resultados de aplicación del algoritmo de clustering $K$-means sobre los datos de la sesión de prueba realizada.

Figura 9

Clustering sesión de prueba

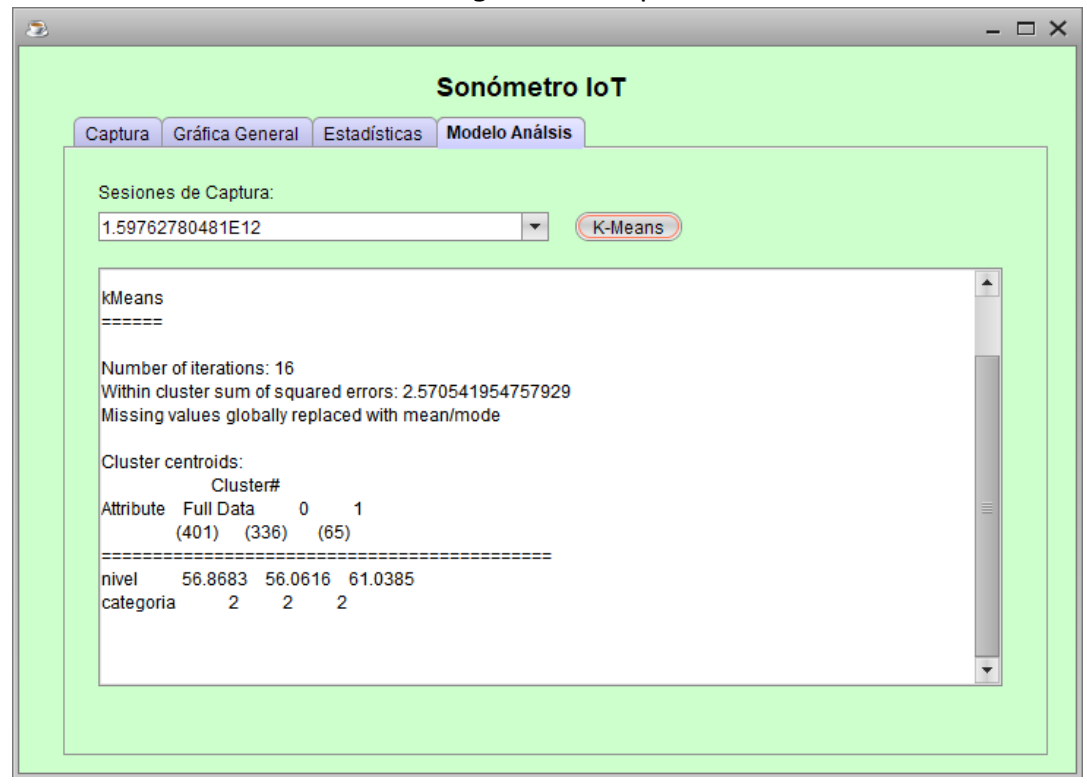

Fuente: Elaboración propia 
Se puede apreciar en la Figura 9 que el modelo obtuvo dos clusters alrededor de los cuales se concentran los datos: $C 1=\{$ nivel=56,06, categoría $="$ considerable" $\}$ y $C 2=\{$ nivel=61,385, categoría="considerable" $\}$. En el caso del cluster C1 se tiene un total de 336 instancias, mientras que en el caso del cluster C2 se tiene un total de 65 instancias. De la misma manera para el total de los datos capturados en la sesión de prueba se obtiene un cluster general Cgeneral=\{nivel=56,858, categoría="considerable" $\}$. Este valor excede levemente la recomendación de la Organización Mundial de la Salud de no superar los 55 dBA durante la noche.

\section{Conclusiones}

En este artículo se propuso como aporte un sistema loT para el seguimiento y análisis de niveles de ruido, el cual toma en consideración las cuatro capas de la arquitectura convencional de loT: captura, almacenamiento, análisis y visualización. El sistema propuesto es una alternativa a los problemas de los equipos de medición convencionales, de tal modo que permite solventar el almacenamiento limitado, da soporte al seguimiento remoto y posibilita la aplicación de algoritmos de aprendizaje supervisado y no supervisado.

La ventaja de este sistema es que permite el aprovechamiento de la precisión de los dispositivos comerciales de medición de niveles de ruido o también conocidos como sonómetros. Lo anterior, gracias a las prestaciones provistas por la librería Bleak de Python, la cual permite acceder a los datos de los servicios y características de los dispositivos abiertos y comerciales. Resultó fundamental para cada caso la interpretación de la trama de datos hexadecimales capturados a partir de estos dispositivos.

Las herramientas y tecnologías escogidas en el desarrollo del sistema demostraron ser adecuadas para dar cumplimiento a los requisitos y/o funcionalidades de cada una de las cuatro capas de la arquitectura loT. A pesar de lo anterior, dado que en el presente caso se hizo uso de una solución híbrida que combina el lenguaje Python con el lenguaje Java, otra de las posibles opciones es usar por completo el lenguaje Python para el desarrollo teniendo en cuenta la librería scikit-learn para el análisis de datos y la librería matplotlib para la visualización de los datos.

La prueba de verificación desarrollada permitió observar que, durante la prueba realizada en un edificio residencial de la ciudad de Cartagena, se obtuvieron valores superiores levemente a la recomendación de la OMS de $55 d B A$ en horas nocturnas. Aunque la prueba tuvo por objetivo verificar la funcionalidad del sistema, a partir de los resultados se puede evidenciar la utilidad en la conducción de estudios de auditoria acústica en diferentes sectores de una ciudad y en diferentes horas del día.

Como trabajo futuro derivado de la presente investigación, se pretende la vinculación de otros algoritmos de clustering al sistema loT tales como DBSCAN. Así mismo se pretende la integración de otros dispositivos de medición de variables ambientales tales como: intensidad lumínica, velocidad del viento, temperatura, humedad relativa del aire, ondas electromagnéticas, entre otros.

\section{Referencias bibliográficas}

Basner, M., Babisch, W., Davis, A., Brink, M., Clark, C., Janssen, S., \& Stansfeld, S. (2014). Auditory and nonauditory effects of noise on health. In The Lancet. https://doi.org/10.1016/S0140-6736(13)61613-X

Begou, P., Kassomenos, P., \& Kelessis, A. (2020a). Dataset on the road traffic noise measurements in the municipality of Thessaloniki , Greece. Data in Brief, 29, 105214. https://doi.org/10.1016/j.dib.2020.105214

Begou, P., Kassomenos, P., \& Kelessis, A. (2020b). Effects of road traffic noise on the prevalence of cardiovascular diseases: The case of Thessaloniki, Greece. Science of the Total Environment. https://doi.org/10.1016/j.scitotenv.2019.134477 
Hernández, L., \& Barriere, L. R. (2017). Diseño de un Monitor de Parámetros Ambientales para Unidades de Cuidados Intensivos Neonatales. XL CONGRESO NACIONAL DE INGENIERÍA BIOMÉDICA, 95-98. https://memorias.somib.org.mx/index.php/memorias/article/view/222

Hueso, M. C., Giménez, A., Sancho, S., \& Gaja, E. (2017). Measurement techniques of noise level in various urban scenarios . Day selection and representative period. Applied Acoustics, 116, 216-228. https://doi.org/10.1016/j.apacoust.2016.09.033

Khan, J., Kakosimos, K., Jensen, S. S., Hertel, O., Sørensen, M., Gulliver, J., \& Ketzel, M. (2020). The spatial relationship between traffic-related air pollution and noise in two Danish cities: Implications for healthrelated studies. Science of the Total Environment. https://doi.org/10.1016/j.scitotenv.2020.138577

Khan, J., Ketzel, M., Kakosimos, K., Sørensen, M., \& Jensen, S. S. (2018). Road traffic air and noise pollution exposure assessment $-A$ review of tools and techniques. In Science of the Total Environment. https://doi.org/10.1016/j.scitotenv.2018.03.374

Leiva, O., \& Higuera, G. (2019). Monitoreo de ruido en sitios cerrados. Visión Electrónica, 1-21.

Marouf, S., Bell, M. C., Goodman, P., Namdeo, A., \& Neasham, J. (2018). Pervasive wireless sensors: A new monitoring tool for road traffic noise evaluation. Applied Acoustics. https://doi.org/10.1016/j.apacoust.2018.01.017

Marouf, S. S., Bell, M. C., Goodman, P. S., Neasham, J., Neasham, J., \& Namdeo, A. K. (2020). Comprehensive study of the response of inexpensive low energy wireless sensors for traffic noise monitoring. Applied Acoustics. https://doi.org/10.1016/j.apacoust.2020.107451

Montes González, D., Barrigón Morillas, J. M., Rey Gozalo, G., \& Godinho, L. (2020). Effect of parking lanes on assessing the impact of road traffic noise on building façades. Environmental Research. https://doi.org/10.1016/j.envres.2020.109299

Neitzel, R. L., Heikkinen, M. S. A., Williams, C. C., Marie, S., \& Dellarco, M. (2016). Pilot study of methods and equipment for in-home noise level measurements. APPLIED ACOUSTICS, 102, 1-11. https://doi.org/10.1016/j.apacoust.2015.08.018

Nurjannah, I., Harijanto, A., \& Supriadi, B. (2017). Sound Intensity Measuring Instrument Based on Arduino Board with Data Logger System. International Journal of Advanced Engineering Research and Science (IJAERS), 4(9), 27-35. https://doi.org/10.22161/ijaers.4.9.7

Peng, J., Zhang, H., \& Wang, D. (2018). Measurement and analysis of teaching and background noise level in classrooms of Chinese elementary schools. Applied Acoustics, 131(June 2017), 1-4. https://doi.org/10.1016/j.apacoust.2017.10.012

Quiroga, E., Jaramillo, S., Campo, W., \& Chanchí, G. (2017). Propuesta de una Arquitectura para Agricultura de Precisión Soportada en loT Proposal of an Architecture for Precision Agriculture Supported in IoT. RISTI: Revista Ibérica de Sistemas e Tecnologias de Informação, 24, 39-56. https://doi.org/10.17013/risti.24.3956

Recio, A., Linares, C., \& Díaz, J. (2018). System dynamics for predicting the impact of traffic noise on cardiovascular mortality in Madrid. Environmental Research, 167(June), 499-505. https://doi.org/10.1016/j.envres.2018.07.021 
Ruparel, P. N., Mistry, N. J., \& Jariwala, N. D. (2013). International Conference on Innovations in Engineering and Technology (ICIET'2013). Health Impacts of Urban Road Traffic Noise, 259-261.

https://doi.org/http://dx.doi.org/10.15242/IIE.E1213578

Senthilkumar, R., Venkatakrishnan, P., \& Balaji, N. (2020). INTELLIGENT BASED NOVEL EMBEDDED SYSTEM BASED IOT. Microprocessors and Microsystems, 103172. https://doi.org/10.1016/j.micpro.2020.103172

Shakya, K. M., Kremer, P., Henderson, K., McMahon, M., Peltier, R. E., Bromberg, S., \& Stewart, J. (2019). Mobile monitoring of air and noise pollution in Philadelphia neighborhoods during summer 2017. Environmental Pollution. https://doi.org/10.1016/j.envpol.2019.113195

Sieber, C., Ragettli, M. S., Brink, M., Olaniyan, T., Baatjies, R., Saucy, A., Vienneau, D., Probst-Hensch, N., Dalvie, M. A., \& Röösli, M. (2018). Comparison of sensitivity and annoyance to road traffic and community noise between a South African and a Swiss population sample. Environmental Pollution. https://doi.org/10.1016/j.envpol.2018.06.007

Stoilova, K., Stoilov, T., \& Abouaïssa, H. (2013). Traffic Lights Optimization with Measurements of Noise Levels. IFAC Proceedings, 46(25), 31-36. https://doi.org/10.3182/20130916-2-TR-4042.00019

Suárez López, D., Morales Espinosa, R., Cordero Gutierrez, I., \& Schreiner de Oliveira, L. (2017). Diseño de una herramienta de medición de ruidos basados en tecnologías Arduino-Rasperry PI. Producción + Limpia, 12(1), 81-87. https://doi.org/10.22507/pml.v12n1a8

Thacher, J. D., Hvidtfeldt, U. A., Poulsen, A. H., Raaschou-Nielsen, O., Ketzel, M., Brandt, J., Jensen, S. S., Overvad, K., Tjønneland, A., Münzel, T., \& Sørensen, M. (2020). Long-term residential road traffic noise and mortality in a Danish cohort. Environmental Research. https://doi.org/10.1016/j.envres.2020.109633

World Health Organization. (2018). World Health Organization. Environmental Noise Guidelines for the European Region.

Zambrano-Monserrate, M. A., \& Ruano, M. A. (2019). Does environmental noise affect housing rental prices in developing countries? Evidence from Ecuador. Land Use Policy. https://doi.org/10.1016/j.landusepol.2019.104059

Esta obra está bajo una Licencia Creative Commons Attribución-NoCommercial 4.0 International

(cc) BY-NC 